\title{
Wind-farm layout optimisation using a hybrid Jensen-LES approach
}

\author{
Vahid S. Bokharaie, Pieter Bauweraerts, and Johan Meyers \\ KU Leuven, Department of Mechanical Engineering, Celestijnenlaan 300A, B3001 Leuven, Belgium \\ Correspondence to: Johan Meyers (johan.meyers@kuleuven.be)
}

Received: 28 April 2016 - Published in Wind Energ. Sci. Discuss.: 11 May 2016

Revised: 29 September 2016 - Accepted: 27 October 2016 - Published: 5 December 2016

\begin{abstract}
Given a wind farm with known dimensions and number of wind turbines, we try to find the optimum positioning of wind turbines that maximises wind-farm energy production. In practice, given that optimisation has to be performed for many wind directions, and taking into account the yearly wind distribution, such an optimisation is computationally only feasible using fast engineering wake models such as the Jensen model. These models are known to have accuracy issues, in particular since their representation of wake interaction is very simple. In the present work, we propose an optimisation approach that is based on a hybrid combination of large-eddy simulation (LES) and the Jensen model; in this approach, optimisation is mainly performed using the Jensen model, and LES is used at a few points only during optimisation for online tuning of the wake-expansion coefficient in the Jensen model, as well as for validation of the results. An optimisation case study is considered, in which the placement of 30 turbines in a $4 \mathrm{~km}$ by $3 \mathrm{~km}$ rectangular domain is optimised in a neutral atmospheric boundary layer. Optimisation for both a single wind direction and multiple wind directions is discussed.
\end{abstract}

\section{Introduction}

Wind turbines are often clustered together in wind farms to save the cost of land and cabling. However, aerodynamic interactions between the turbines in the form of so-called wakes (low-speed regions) that form behind wind turbines lead to power reductions in "waked" turbines of up to $50 \%$ compared to a lone-standing wind turbine in undisturbed flow (Barthelmie et al., 2010). These interactions are very important when considering the topological placement of wind turbines in large wind farms.

In order to optimally design wind-farm layout, models are necessary that accurately predict the aerodynamic turbinewake interaction effects. Such models need to be very fast, as wind-farm design optimisation needs to consider the full spectrum of wind directions over a wind farm's operational lifetime, thus requiring many thousands of model evaluations. Moreover, wind-farm design is a multidisciplinary problem in which the aerodynamic wake-interaction model is only one of the models, next to turbine load models, lifetime analysis, economic investment models, etc. (see, e.g., Zaaijer, 2013). Today, the wake model that is most used is the Jensen model (Jensen, 1983; Katic et al., 1986). It is a simple and fast model, but it is known to be inaccurate when looking at individual power predictions of turbines in various waked conditions (Barthelmie et al., 2009; Gaumond et al., 2014; Niayifar and Porté-Agel, 2015). Layout optimisation of wind farms using fast wake models has been investigated in numerous studies (Marmidis et al., 2008; Emami and Noghreh, 2010; Kusiak and Song, 2010; González et al., 2010; Saavedra-Moreno et al., 2011; Du Pont and Cagan, 2012; Chowdhury et al., 2012; Samorani, 2013; Chen et al., $2013 b)$. However, the accuracy of such optimisation results has always remained a concern in view of the limited reliability of wake models, and this has recently led to a renewed interest in the formulation of accurate but fast wake models (Stevens et al., 2015; Niayifar and Porté-Agel, 2015).

In the last five years, the detailed simulation of wind-farmatmospheric-boundary-layer interaction and turbine wake interactions based on high-fidelity simulation tools such as large-eddy simulation (LES) have become very popular (see, e.g., Meyers and Meneveau, 2010; Calaf et al., 2010; Yang et al., 2012; Meyers and Meneveau, 2013; Wu and Porté-Agel, 
2013; Allaerts and Meyers, 2015), leading to many new insights into the flow physics of wind farms. Given known and constant meteorological conditions, these types of models provide a detailed time-resolved prediction of the turbulent flow in a wind farm with resolution of spatial flow structures in the order of $20 \mathrm{~m}$ and temporal fluctuations in the order of $10 \mathrm{~s}$. Although it is computationally infeasible in LES of wind farms to resolve all the detailed flow physics, such as the turbine blade-boundary layers (with length scale below a millimetre), these models do lead to quite accurate predictions of wakes and wake merging when compared to windtunnel and field experiments (Porté-Agel et al., 2011; Wu and Porté-Agel, 2013; Munters et al., 2016a). Unfortunately, LES of wind farms requires supercomputing and simulation times that are several hours to days for one single atmospheric condition. Hence, these models are not useful for layout optimisation purposes.

In the current work, we investigate a hybrid approach in which the Jensen model is used during optimisation, but we use LES to gradually adapt the Jensen model and verify the optimisation results. To this end, the wake-expansion coefficient in the Jensen model is iteratively fitted based on LES. In itself, tuning of the wake-expansion coefficient (e.g. to experiments) is quite common, but it is well known that the coefficient depends on atmospheric conditions and farm layout, and it may also best depend on streamwise distance into the farm (Stevens et al., 2015). Therefore, a coefficient that is tuned a priori will not fit all possible scenarios that are encountered during layout optimisation of a wind farm over its relevant range of atmospheric conditions. In a hybrid JensenLES approach, it is possible to adapt the coefficient a posteriori during optimisation depending on layout, wind direction, etc. The main focus of the current work is on the formulation of an approach that is computationally feasible, given the very high costs of performing LES (even in a hybrid JensenLES optimisation). We demonstrate the proposed methodology on a moderately sized wind farm of 30 turbines in a $4 \mathrm{~km}$ by $3 \mathrm{~km}$ farm area.

This paper is organised as follows. In Sect. 2 the mathematical formulation for the optimisation problem is stated, and the simulation models (both Jensen and LES) and the optimisation methodology are introduced. In Sect. 3, results are presented. First, the different steps in the algorithm are highlighted for a single wind-direction optimisation case in Sect. 3.1-3.3. Subsequently, in Sect. 3.4, some results for optimisation with multiple wind directions are discussed. Finally, conclusions are presented in Sect. 4.

\section{Problem description and methodology}

In Sect. 2.1, the optimisation problem description is introduced. Subsequently, the Jensen model is briefly reviewed in Sect. 2.2. The LES simulation environment is discussed in
Sect. 2.3, and finally the hybrid Jensen-LES approach and the optimisation method are presented in Sect. 2.4.

\subsection{Problem description}

Consider a set of $N_{\mathrm{t}}$ turbines that are to be placed in a fixed domain $\Omega$. Given constant atmospheric conditions and wind direction (parameterised in a vector $\boldsymbol{\mu}$ ), the average power output of a turbine at position $\boldsymbol{x}_{i}$ in the wind farm is

$\bar{P}_{i}\left(\boldsymbol{x}_{i}, \boldsymbol{\mu}\right)=\frac{1}{T} \int_{0}^{T} P_{i}\left(\boldsymbol{x}_{i}, t, \boldsymbol{\mu}\right) \mathrm{d} t$,

where $P_{i}\left(\boldsymbol{x}_{i}, t, \boldsymbol{\mu}\right)$ corresponds to the instantaneous power output of the turbine (given atmospheric conditions $\mu$ ), which is subject to turbulent wind fluctuations, and $T$ is a time averaging window that is sufficiently long to average out the turbulence effects. Note that the Jensen model (see Sect. 2.2) directly predicts $\bar{P}_{i}$ of turbines in a wind farm, while, for example, experimental measurements as well as results from LES (see Sect. 2.3) yield $P_{i}\left(\boldsymbol{x}_{i}, t, \boldsymbol{\mu}\right)$ and thus explicitly require the above time averaging.

The optimisation problem that we consider is formulated as follows:

$$
\begin{array}{cl}
\underset{\boldsymbol{x}_{i}}{\operatorname{maximise}} & \int \sum_{i=1}^{N_{\mathrm{t}}} \bar{P}_{i}\left(\boldsymbol{x}_{i}, \boldsymbol{\mu}\right) f_{\mathrm{p}}(\boldsymbol{\mu}) \mathrm{d} \boldsymbol{\mu} \\
\text { subject to } & \boldsymbol{x}_{i} \in \Omega, \quad \forall i \in\left\{1, \cdots, N_{\mathrm{t}}\right\} \\
& \left\|\boldsymbol{x}_{i}-\boldsymbol{x}_{j}\right\|_{2} \geq d_{\min } \\
& \forall i, j \in\left\{1, \cdots, N_{\mathrm{t}}\right\}, i \neq j,
\end{array}
$$

where $\Omega$ is the wind-farm domain in which turbines can be freely placed and $f_{\mathrm{p}}(\boldsymbol{\mu})$ is the joint probability density function of atmospheric conditions $\boldsymbol{\mu}$ over which optimisation needs to be carried out (e.g. the yearly wind-direction distribution, atmospheric stability class). Finally, $d_{\min }$ is a constraint on the minimum distance between turbines. In theory, the minimum distance between turbines is $1.0 D$ (with $D$ the rotor diameter). In the current study, we will consider a $d_{\text {min }}$ of $2.0 \mathrm{D}$ for all optimisation cases.

The solution of the above optimisation problem requires a model for $\bar{P}_{i}\left(\boldsymbol{x}_{i}, \boldsymbol{\mu}\right)$. This is discussed next in Sect. 2.2 for the Jensen model and in Sect. 2.3 for the LES model. To solve the above optimisation problem, we use the cross-entropy optimisation method (De Boer et al., 2005; Rubinstein, 1999) in combination with a hybrid Jensen-LES model as discussed in Sect. 2.4. Finally, note that, for ease of notation, we drop $\boldsymbol{\mu}$ as an argument in $\bar{P}_{i}$. In fact, the conditions $\boldsymbol{\mu}$ (e.g. wind direction, turbulence intensity) are implicity contained on the set-up and boundary conditions of the respective models below.

\subsection{The Jensen wake model}

We briefly review the Jensen wake model as originally developed by Jensen (1983) and Katic et al. (1986). 
The model commences by assuming that each turbine generates a radially and azimuthally uniform wake that linearly expands with downstream distance from the turbine. Using simple mass conservation, this allows the velocity deficit generated by turbine $i$ to be described as

$\Delta U_{i}\left(s_{i}\right)=U_{\infty} \frac{1-\sqrt{1-C_{\mathrm{T}, i}}}{\left(1+k_{\mathrm{w}} s_{i} / R\right)^{2}}, \quad s_{i}>0$,

where $C_{\mathrm{T}, i}$ is the turbine thrust coefficient and $s_{i}=\left(\boldsymbol{x}-\boldsymbol{x}_{i}\right)$. $\boldsymbol{e}_{\mathrm{f}}$ is the downstream axial distance from the turbine, and $\boldsymbol{e}_{\mathrm{f}}$ the unit vector in the mean-flow direction. Obviously, $s_{i}>0$. Upstream of a turbine, its own generated wake has a velocity deficit $\Delta U_{i}=0$. Furthermore, $k_{\mathrm{W}}$ is the linear wakeexpansion coefficient, and $R$ is the rotor radius. Correlations exist that relate $k_{\mathrm{W}}$ to the incoming atmospheric boundary layer; for example (Lissaman, 1979; Frandsen, 1992)

$k_{\mathrm{W}}=\frac{u_{*}}{U_{\infty}}=\frac{\kappa}{\ln \left(z_{\mathrm{h}} / z_{0}\right)}$

is commonly used, with $\kappa$ the von Kármán constant, $z_{\mathrm{h}}$ the turbine hub height, and $z_{0}$ and $u_{*}$ the surface roughness and friction velocity of the incoming atmospheric boundary layer. Note that, in the current study, we will use LES to adapt $k_{\mathrm{w}}$ in our optimisation procedure as discussed in Sect. 2.4. Finally note that the wake expansion is vertically restricted by the ground once the wake radius grows larger than the turbine hub height. However, the ground is not directly modelled, but instead mirror turbines are added below the ground, with wakes that are included in the wake-merging model (Lissaman, 1979) (see below).

In order to estimate the power output $\bar{P}_{i}$, the turbine's incoming mean velocity is required. It is modelled as $U_{i \text {,in }}=$ $U_{\infty}-\Delta U_{i, \text { in }}$, with $U_{\infty}$ the wind-farm inflow velocity at hub height and $\Delta U_{i \text {,in }}$ the upstream velocity deficit experienced by turbine $i$. The deficit $\Delta U_{i \text {,in }}$ is heuristically modelled by quadratically adding upstream wake deficits as follows:

$\Delta U_{i, \text { in }}=\left[\sum_{j \in \mathcal{S}_{i}}\left(\Delta U_{j}\left(s_{i j}\right)\right)^{2}\right]^{1 / 2}$.

Here $\mathcal{S}_{i}$ is the set of all upstream turbines that have a wake that geometrically intersects with turbine $i$ and $s_{i j}$ is the distance along the wind direction between turbine $i$ and $j$. In order to include the effect of the ground on wake development, mirror turbines (below the ground) are added to the set $\mathcal{S}_{i}$ for each turbine whose wake is restricted by the ground. It is furthermore possible that wakes only partially overlap, in which case the rotor area of the inflow turbine is split into regions with different overlaps. More details on the approach can be found in Rathmann et al. (2007).

Once the turbine inflow velocities $U_{i \text {, in }}$ are determined, the power per turbine is calculated as

$\bar{P}_{i}\left(x_{i}\right)=\frac{1}{2} C_{\mathrm{P}, i} \rho U_{i, \text { in }}^{3}$, where $C_{\mathrm{P}, i}$ is the wind turbine's power coefficient. For an ideal turbine, $C_{\mathrm{P}, i}$ follows from axial momentum theory from, i.e.

$C_{\mathrm{P}, i}=\frac{1}{2} C_{\mathrm{T}, i}\left[1-\left(1-C_{\mathrm{T}, i}\right)^{1 / 2}\right]$.

For a real turbine, $C_{\mathrm{P}, i}$ can be expressed as a function of $C_{\mathrm{T}, i}$ and wind speed, using either a mapping specific to the turbine or blade-element momentum theory, and this can be straightforwardly used in the Jensen model. In the current study, we will simply use above ideal relationship, as our main focus is on the development and demonstration of the hybrid JensenLES approach, and not so much on the specifics of the selected turbine model.

\subsection{Large-eddy simulation environment and simulation set-up}

Simulations are performed using SP-Wind, developed at KU Leuven (Meyers and Meneveau, 2010, 2013; Allaerts and Meyers, 2015; Goit and Meyers, 2015; Munters et al., 2016a). SP-Wind solves the filtered incompressible NavierStokes equations, which are given by

$$
\begin{aligned}
\nabla \cdot \widetilde{\boldsymbol{u}} & =0 \\
\frac{\partial \widetilde{\boldsymbol{u}}}{\partial t}+\widetilde{\boldsymbol{u}} \cdot \nabla \widetilde{\boldsymbol{u}} & =-\frac{1}{\rho} \nabla \widetilde{p}+\nabla \cdot \boldsymbol{\tau}_{\mathrm{M}}-\boldsymbol{f},
\end{aligned}
$$

where $\widetilde{\boldsymbol{u}}(\boldsymbol{x}, t)=\left[\widetilde{u}_{1}, \widetilde{u}_{2}, \widetilde{u}_{3}\right]$ is the resolved velocity field, $\widetilde{p}$ is the pressure field, and $\tau_{M}$ is the sub-grid-scale (SGS) model. We use a standard Smagorinsky model (Smagorinsky, 1963) with Mason and Thomson's wall damping (Mason and Thomson, 1992) to model the SGS stress. Furthermore, $-\boldsymbol{f}$ represents the forces (per unit mass) introduced by the turbines on the flow. In LES of wind-farm boundary layers, this turbine-induced force is commonly modelled using an actuator-disc model (ADM), as full meshing of the turbine blades and geometry leads to computational grids that are too large for current-day computers. Expressed for turbine $i$, this force corresponds to (Meyers and Meneveau, 2010; Goit and Meyers, 2015):

$\boldsymbol{f}^{(i)}=\frac{1}{2} C_{\mathrm{T}, i}^{\prime} \widehat{V}_{i}^{2} \mathscr{R}_{i}(\boldsymbol{x}) \boldsymbol{e}_{\perp} ; \quad i=1 \cdots N_{\mathrm{t}}$,

where $\boldsymbol{e}_{\perp}$ represents the unit vector perpendicular to the turbine disc, and $\mathscr{R}_{i}(\boldsymbol{x})$ is a geometrical smoothing function that distributes the uniform surface force of the turbine over surrounding LES grid cells, with $\int_{\Omega} \mathscr{R}_{i}(\boldsymbol{x}) \mathrm{d} \boldsymbol{x}^{\prime}=A$ and $A$ the turbine disc area. Moreover, $\widehat{V}_{i}$ is the disc-averaged turbine velocity, and $C_{\mathrm{T}, i}^{\prime}$ is the disc-based thrust coefficient. Unlike the conventional thrust coefficient $C_{\mathrm{T}}$ (used in the Jensen model), which is based on undisturbed velocity far upstream of a turbine, $C_{\mathrm{T}, i}^{\prime}$ is defined using the velocity at the turbine disc. It results from integrating lift and drag coefficients over the turbine blades, taking design geometry and 
flow angles into account (see Appendix A in Goit and Meyers, 2015, for a detailed formulation). Based on axial momentum theory, we have (Calaf et al., 2010)

$C_{\mathrm{T}}=\frac{C_{\mathrm{T}}^{\prime}}{\left(1+C_{\mathrm{T}}^{\prime} / 4\right)^{2}}$

which provides a direct relation between the thrust coefficient used in the Jensen model and the disc-based thrust coefficient used in the LES model. Finally, given the velocity field $\widetilde{\boldsymbol{u}}(\boldsymbol{x}, t)$ from a LES, the average power output for turbine $i$ is determined from

$\bar{P}_{i}\left(\boldsymbol{x}_{i}\right)=\frac{1}{T} \int_{0}^{T} \iiint f^{(i)} \cdot \widetilde{\boldsymbol{u}} \mathrm{d} x \mathrm{~d} t$.

In Fig. 1 a typical snapshot of a horizontal velocity field $\widetilde{u}_{1}(\boldsymbol{x}, t)$ is shown, including an outline of the simulation domain that is considered in the current study. The main domain size is $L_{y} \times L_{x} \times L_{z}=8.0 \times 6.0 \times 1.0 \mathrm{~km}^{3}$, where $x$ is always the main flow direction and $z$ is the vertical direction. The wind farm is inserted in a subdomain $\Omega=$ $4.0 \mathrm{~km} \times 3.0 \mathrm{~km}$ (also marked on the figure). At $z=0$ a classical high-Reynolds-number wall-stress boundary condition is used (Moeng, 1984; Bou-Zeid et al., 2005), which is parameterised by the ground surface roughness $z_{0}$, for which we use $z_{0}=0.1 \mathrm{~m}$. At $z=L_{z}$ a symmetry condition is used, and in the $y$ direction periodic boundary conditions are used. Finally, at $x=0$ an inflow boundary condition is used.

The inflow is generated in a separate precursor simulation (also shown in Fig. 1), which employs shifted periodic boundary conditions to avoid artificial spanwise locking of the typical low-speed streaks observed in boundary layers (see Munters et al., 2016b, for details). For the precursor simulation, a domain size of $8.0 \times 6.0 \times 1.0 \mathrm{~km}^{3}$ is selected. The precursor simulation is driven by a constant pressure gradient, which corresponds to $\nabla p_{\infty} / \rho=u_{*}^{2} / L_{z}$, where $u_{*}=\left(\tau_{\mathrm{w}} / \rho\right)^{1 / 2}$ is the friction velocity in the precursor domain. In the current work, we are interested in region II operation of wind turbines for which $C_{\mathrm{T}}^{\prime}$ can be presumed to be constant. Given that also $z_{0}$ and $z_{\mathrm{h}}$ are fixed, simulation results remain dynamically equivalent for any selected value of $u_{*}$, with velocity scaling proportionally with $u_{*}$ and time scaling inversely proportionally with $u_{*}$. An output of our precursor simulation (given $z_{0}=0.1 \mathrm{~m}$ ) is the hub-height velocity $u_{\mathrm{h}} \approx 17.5 u_{*}$. Thus, to obtain a realistic region II hubheight velocity of, for example, $8 \mathrm{~m} \mathrm{~s}^{-1}$, it suffices to select $u_{*}=0.457 \mathrm{~m} \mathrm{~s}^{-1}$. However, since all later results and comparisons with the Jensen model are normalised with inflow velocity, or with first-row power output, the exact value of $u_{*}$ is not further important (in our simulations, we just use $\left.u_{*}=1\right)$.

For the discretisation of the governing equations, SP-Wind uses a pseudo-spectral method in the horizontal directions, applying the $3 / 2$ rule for dealiasing (Canuto et al., 1988).
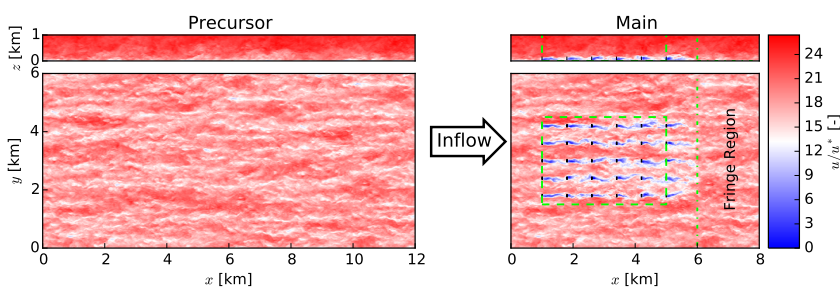

Figure 1. Snapshot of an instantaneous velocity field in the precursor domain and main simulation domain obtained from SP-Wind. Left panels: precursor, with side view (top) and plan view (bottom). Right panels: main, with side view (top) and plan view (bottom). Wind-farm area $\Omega$ is shown in the green dashed box, and the fringe region with the green dash-dot line.

In the vertical direction, a fourth-order energy-conservative finite-difference discretisation scheme is used (Verstappen and Veldman, 2003). Non-periodic boundary conditions in the $x$ direction are implemented using a fringe-region technique, with a fringe region located in the last $2 \mathrm{~km}$ of the domain (for details, see Spalart and Watmuff, 1993; Stevens et al., 2014; Munters et al., 2016a, b). Mass is conserved by using a Poisson equation for the pressure, which is solved using a direct solver. Finally, time integration is performed using a classical four-stage fourth-order Runge-Kutta scheme. For the simulations discussed in this paper, a fixed time step of $0.4 \mathrm{~s}$ corresponding to a Courant-Friedrichs-Lewy (CFL) number of approximately 0.4 is used. The computational grid for the main domain corresponds to $N_{y} \times N_{x} \times N_{z}=$ $256 \times 256 \times 80$; this is also the case for the precursor domain. For nonlinear operations we use the $3 / 2$ dealiasing rule, so that all nonlinear operations in real space are performed on $384 \times 384 \times 80$ grids for both domains. Simulation parameters are summarised in Table 1.

In the current study, we consider a rectangular fixed windfarm domain $\Omega$ of $4.0 \mathrm{~km}$ by $3.0 \mathrm{~km}$ (see above), in which 30 turbines are to be optimally placed. We take generic wind turbines with a diameter of $D=100 \mathrm{~m}$ and hub height of $z_{\mathrm{h}}=100 \mathrm{~m}$ each. The selected disc-based and standard thrust coefficients correspond to $C_{\mathrm{T}}^{\prime}=2.0$ and $C_{\mathrm{T}}=8 / 9$ respectively. The choice of turbines, simulation domain, and selected computational grids corresponds to the typical case set-ups found in Calaf et al. (2010) and Meyers and Meneveau (2013), and we refer the reader to these studies for detailed grid sensitivity analysis, for example.

Finally, simulations are initialised by first performing a spin-up of the turbulence in the precursor simulation. Starting from a logarithmic mean profile with random perturbations, the precursor simulation is advanced in time for $15000 / u_{*}$ s so that realistic turbulence can develop. Subsequently, for every wind-farm layout, the precursor and main domain are run simultaneously, and an additional spin-up period of $2000 / u_{*} \mathrm{~s}$ is simulated. This corresponds to at least $5 u_{*}$ flow-through times of the main domain. At this point in time, time averaging of LES results is started. 


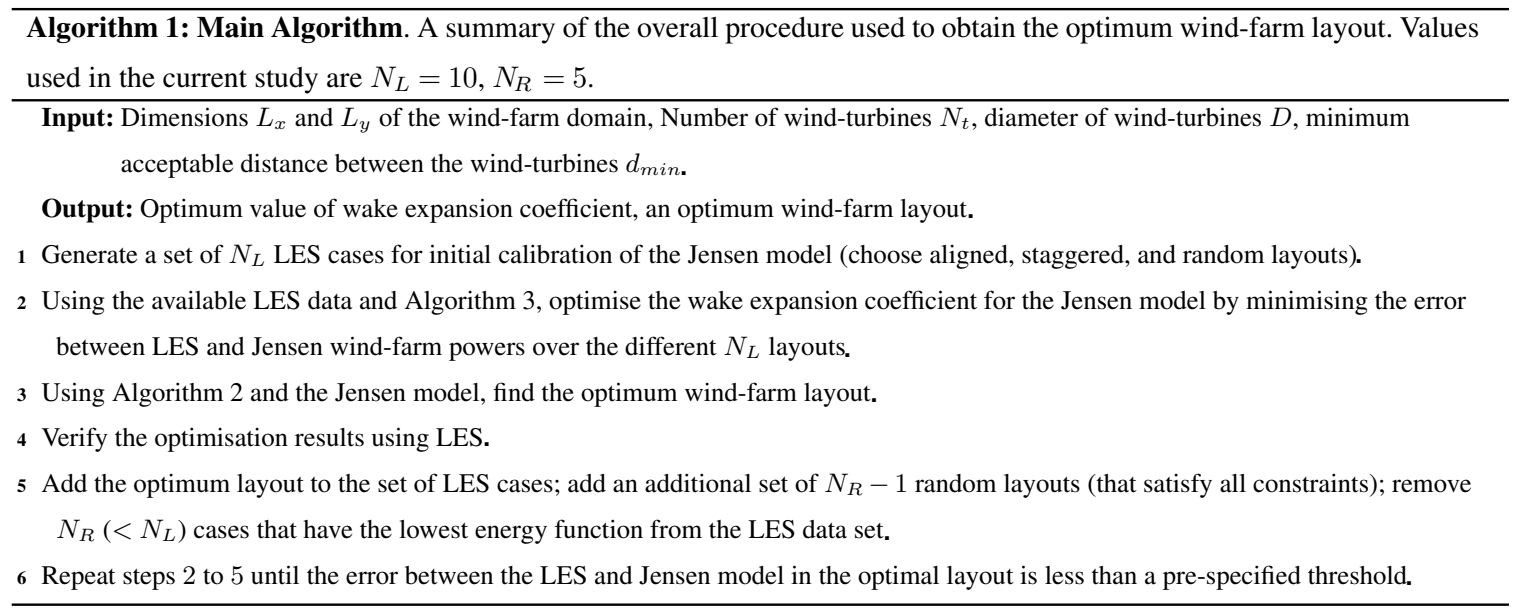

Table 1. Simulation parameters. Results remain dynamically equivalent for any selected value of the friction velocity $u_{*}$. The hubheight velocity obtained in the precursor simulation corresponds to $u_{\mathrm{h}} \approx 17.5 u_{*}$.

\begin{tabular}{ll}
\hline $\begin{array}{l}\text { Total domain size (with } \\
\text { fringe region) } \\
\text { Total domain size } \\
\text { (without fringe region) }\end{array}$ & $8 \mathrm{~km} \times 6 \mathrm{~km} \times 1 \mathrm{~km}$ \\
$\begin{array}{l}\text { Optimisation domain size } \\
\text { Turbine diameter }\end{array}$ & $4 \mathrm{~km} \times 3 \mathrm{~km} \times 1 \mathrm{~km}$ \\
Turbine height & $100 \mathrm{~m}$ \\
Driving pressure & $100 \mathrm{~m}$ \\
gradient (precursor) & $-u_{*}^{2} / 1000 \mathrm{~m} \mathrm{~s}^{-2}$ \\
Surface roughness & $0.1 \mathrm{~m}$ \\
Grid size & $256 \times 256 \times 80$ \\
Cell size & $31.25 \mathrm{~m} \times 23.44 \mathrm{~m} \times 12.5 \mathrm{~m}$ \\
Time step & $0.4 / u_{*} \mathrm{~s}$ \\
\hline
\end{tabular}

In Fig. 2, a detailed convergence analysis of the farm power and the power output of a single turbine is shown for an aligned wind-farm layout (corresponding to Case 4 in Table 2 below). In Fig. 2a, a power histogram is shown for the wind farm, as well as for two individual turbines in the farm. Figure $2 \mathrm{~b}$ shows results of the relative error $\epsilon_{P}$ of the time average as a function of the averaging time $T$ (see Eq. 1), where

$\epsilon_{P}(T)=\frac{\frac{1}{T} \int_{0}^{T} P(t) \mathrm{d} t-\bar{P}_{\text {ref }}}{\bar{P}_{\text {ref }}}$.

For reference $\bar{P}_{\text {ref }}$ we use an average obtained over a period of $40 / u_{*} \mathrm{~h}$ (with $u_{*}=0.457 \mathrm{~m} \mathrm{~s}^{-1}$ taken as a realistic value, this corresponds to averaging over $88 \mathrm{~h}$ in physical time). It is seen from the figure that, for limited averaging times, errors can be quite significant, in particular when looking at the single-turbine average. In fact, it is well known that the time average in turbulent flows converges as $T^{-1 / 2}$ (see, e.g., Tennekes and Lumley, 1972). This is also seen in
Fig. 2b: errors decrease fast at low values of $T$, but afterwards convergence stagnates. This is particularly problematic when looking at the turbine average power, which requires roughly 15 to $20 / u_{*}$ h to converge within $1 \%$ of the reference average (requiring excessive computational costs - see below). It is further seen that the error on the overall wind-farm power converges significantly faster, i.e. an error of $1 \%$ is reached after approximately $5 / u_{*} \mathrm{~h}$. This is related to the fact that $N_{\mathrm{t}}$ partly uncorrelated turbine power signals are accumulated. Therefore, in order to limit computational effort related to LES in a hybrid Jensen-LES approach, we will formulate our approach based on matching LES and Jensen farm power levels. In order to avoid overfitting of the Jensen wake-expansion coefficient, we use an ensemble of different wind-farm layouts that gradually evolve during optimisation towards layouts that are more optimal in terms of power extraction. This approach is further discussed in Sect. 2.4.

In terms of computational cost, the spin-up of the precursor simulation is the most expensive (but needs to be done only once), amounting to $32 \mathrm{~h}$ of wall-clock time on the ThinKing cluster of the Flemish Supercomputer Centre, using eight Ivy Bridge nodes consisting of two 10-core "Ivy Bridge" Xeon E5-2680v2 CPUs $(2.8 \mathrm{GHz}, 25 \mathrm{MB}$ level 3 cache) for a total of 160 cores. Wind-farm spin-up takes around $14 \mathrm{~h}$ of wall-clock time on the same processor layout. Subsequent averaging takes around $9 \mathrm{~h}$ of wall-clock time per $3600 / u_{*} \mathrm{~s}$ of wind-farm time. In order to keep overall computational costs under control, we limit time averaging in the current work to $3600 / u_{*} \mathrm{~s}$ (roughly corresponding to at least $9 u_{*}$ flow-through times). This yields an expected error level on the power output of $2 \%$ (see discussion above and Fig. 2b). In practice, for optimisation over a single atmospheric condition $\mu$ (e.g. a single wind direction), it may be advisable to use at least $5 / u_{*} \mathrm{~h}$ for the current case setup. However, when considering optimisation over a range of conditions, the impact of this variability will be further averaged out. 

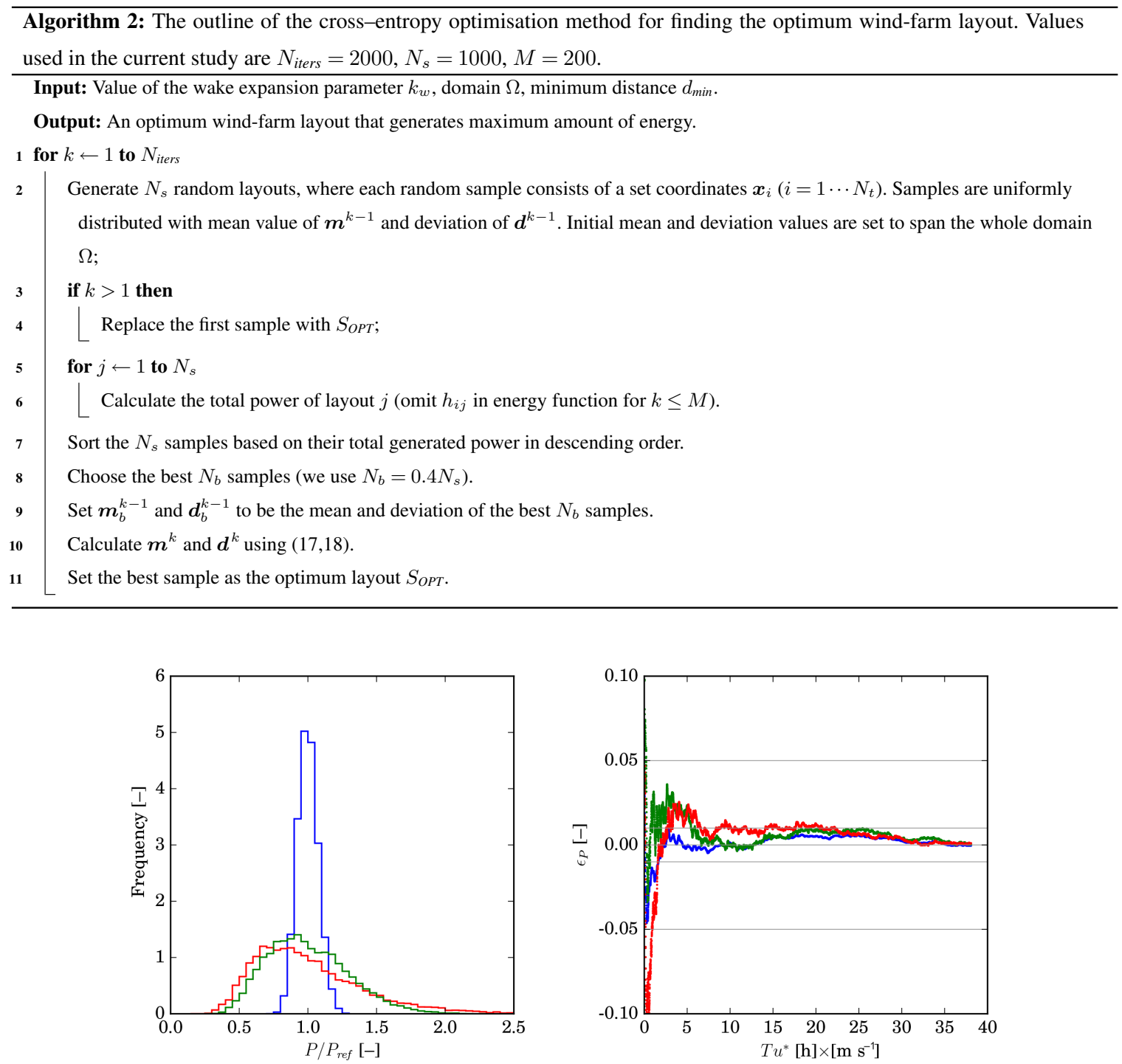

Figure 2. Convergence analysis of wind-farm and turbine power of an aligned wind-farm case (Case 4 in Table 2). (a) Probability density function of wind-farm power output and power output of a front-row and back-row turbine. (b) Convergence error $\epsilon_{P}$ as a function of averaging time $T$ for the wind-farm power, and for the power of a front-row and back-row turbine. Blue line: wind-farm power; green line: first-row turbine; red line: last-row turbine.

\subsection{Hybrid Jensen-LES approach and cross-entropy optimisation}

In the current manuscript, we propose a hybrid Jensen-LES approach for wind-farm layout optimisation. To that end, the layout optimisation is based on the Jensen model, but the wake-expansion coefficient $k_{\mathrm{W}}$ is iteratively used to fit the Jensen model to a set of LES data that is gradually adapted to the layouts that are explored during optimisation. The approach is summarised in Algorithm 2. Here, we describe the approach considering a single atmospheric condition $\boldsymbol{\mu}$ (see Eq. 2), e.g., a single wind direction. Generalisation is straightforward, and optimisation over different wind directions will be discussed in Sect. 3.4.
In a first step, a set of $N_{\mathrm{L}}$ LES cases of regular and random layouts are generated. This set is used to fit $k_{\mathrm{w}}$ using Algorithm 4 (see below). Subsequently, layout optimisation is performed using the Jensen model and Algorithm 3 (see further below). The optimal layout is then added to the set of LES cases, and a number of $N_{\mathrm{R}}-1\left(N_{\mathrm{R}}<N_{\mathrm{L}}\right)$ additional random layouts are added as well. Moreover, the $N_{\mathrm{R}}$ LES cases with lowest generated powers are removed from the set. This new set is used to refit $k_{\mathrm{w}}$, subsequently starting a new layout optimisation. By doing so, the LES data set used for fitting is gradually taking more optimal layouts into account, while layouts that are least optimal are removed from the set. 


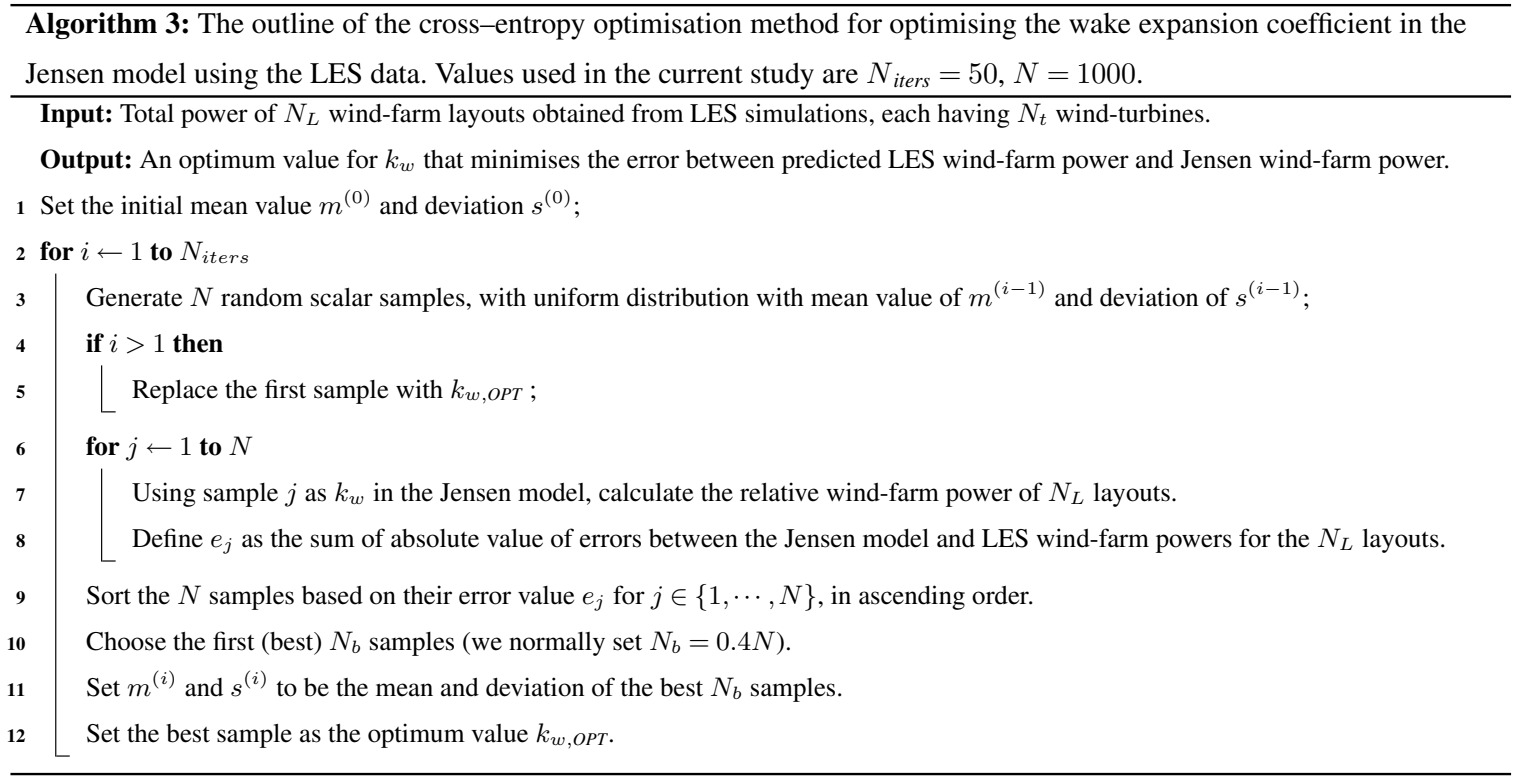

The procedure described above directly uses wind-farm power to fit the wake-expansion coefficient and avoids using errors on individual turbine power output. As discussed in Sect. 2.3, this reduces the need for time averaging in the LES, and significantly lowers computational costs. Moreover, by including $N_{\mathrm{L}}$ different layouts, potential overfitting of $k_{\mathrm{w}}$ is avoided, and the influence of remaining LES convergence errors on the optimal fit is further reduced.

For the layout optimisation in Algorithm 3 and the optimal fit of $k_{\mathrm{w}}$ in Algorithm 4, we employ the cross-entropy (CE) method. This method was originally developed to estimate the probability of rare events. Later on, it was realised that it is also very effective in solving difficult nonconvex optimisation problems. The method is explained in detail by De Boer et al. (2005) and Rubinstein (1999), among others. Here, we briefly review the main features of the approach, as well as further detailing how we use it in a hybrid Jensen-LES optimisation of wind-farm layout. In our hybrid Jensen-LES optimisation approach of wind-farm layout, we use the CE method both for Jensen-based layout optimisation, as well as for the adaptive fitting of the Jensen wakeexpansion coefficient against a range of LES results (as further detailed below). However, it is important to emphasise that any feasible optimisation method may be used for this. For instance, recently, some work has focussed on the use of a gradient-based layout optimisation approach in combination with engineering wake models (Fleming et al., 2016), while others have previously looked into the use of, for example, a particle-swarm method (Wan et al., 2010) and genetic algorithms (Chen et al., 2013a).

First of all, the optimisation problem Eq. (2) is slightly reformulated in order to better cope with the second inequality constraint (as further discussed below, the first constraint is more straightforward to enforce directly). Therefore, we con- sider following a non-smooth problem,

$\max _{\boldsymbol{x}_{i}} \sum_{i=1}^{N_{\mathrm{t}}} \bar{P}_{i}\left(\boldsymbol{x}_{i}\right)+\sum_{i=1}^{N_{\mathrm{t}}} \sum_{j=1}^{i-1} h_{i j}\left(\boldsymbol{x}_{i}, \boldsymbol{x}_{j}\right)$

subject to

$\boldsymbol{x}_{i} \in \Omega, \quad \forall i \in\left\{1, \cdots, N_{\mathrm{t}}\right\}$,

where

$h_{i j}\left(\boldsymbol{x}_{i}, \boldsymbol{x}_{j}\right)=\left\{\begin{aligned}-\infty & \left\|\boldsymbol{x}_{i}-\boldsymbol{x}_{j}\right\|_{2}<d_{\min } \\ 0 & \text { otherwise. }\end{aligned}\right.$

This formulation is fully equivalent to Eq. (2).

The CE method for solving the optimal placement problem now essentially involves three steps. In a first step, a set of $N_{\mathrm{s}}$ uniformly distributed random samples of the optimisation parameters $\boldsymbol{x}_{i}$ are generated with a given mean value $\boldsymbol{m}^{(0)}$ and deviation $\boldsymbol{d}^{(0)}$ (note that both $\boldsymbol{m}$ and $\boldsymbol{d}$ have dimension $2 \times N_{\mathrm{t}}$ ). At startup (iteration 0), no prior knowledge of the optimisation problem is available, so we chose the mean and deviation such that the distribution spans the whole feasible parameter range $\Omega$. In the second step, samples are sorted according to their energy function value. The best $N_{b}<N_{\mathrm{s}}$ samples are chosen, and the mean $\boldsymbol{m}_{\mathrm{b}}^{(k)}$ and deviation $\boldsymbol{d}_{\mathrm{b}}^{(k)}$ of this set (in iteration step $k$ ) is calculated. In a third step, a next generation of samples (iteration step $k+1$ ) is then created using a uniform distribution with a mean and deviation of

$$
\begin{aligned}
\boldsymbol{m}^{(k+1)} & =\boldsymbol{m}^{(k)}+\alpha\left(\boldsymbol{m}_{b}^{(k)}-\boldsymbol{m}^{(k)}\right), \\
\boldsymbol{d}^{(k+1)} & =\boldsymbol{d}^{(k)}+\alpha\left(\boldsymbol{d}_{b}^{(k)}-\boldsymbol{d}^{(k)}\right),
\end{aligned}
$$

where the parameter $\alpha$ is selected in the $[0,1]$ range, specifying how conservative or exploratory the algorithm is. This procedure continues until the end condition is met, which is 
usually set by specifying the maximum number of iterations. We also transfer the optimum value in each generation to the next generation, so that the energy function value of the optimum in each generation increases monotonically.

The treatment of the constraint $\boldsymbol{x}_{i} \in \Omega$ is straightforward. Whenever a turbine location in a sample falls outside $\Omega$, the location is simply orthogonally projected on the boundary of $\Omega$. Note that turbines in samples in the initial generation always fall in $\Omega$, but in later generations, this is not always the case. Though the projection on $\Omega$ will slightly change the distribution, as relatively more sample points can end up on the boundary, we did not find this to hamper the convergence of our algorithm. Finally, the treatment of the distance constraint is implicitly handled by the energy function formulation and does not, in principle, require any further attention.

Given the Jensen model, and an input for the wakeexpansion coefficient $k_{\mathrm{w}}$, the cross-entropy layout optimisation is summarised in Algorithm 2, and specific choices are documented. We run the cross-entropy optimisation scheme for $N_{\text {iter }}=2000$ iterations; however, we find it beneficial for convergence and computational efficiency to omit $h_{i j}$ in the energy function during the first $M$ iterations, and to only enforce the $h_{i j}$ constraint for $k>M$. We take $M=200$ in our implementation.

The standard deviation of samples in the cross entropy scheme eventually converge to zero. Once the standard deviation has become small, and if the algorithm is locked in a local optimum, it will no longer break away from it. To reduce the chance of this happening, we reset the calculated value of $\boldsymbol{d}$ after 1000 iterations. For turbines with $x$ coordinate less than $0.5 \mathrm{~km}$ or bigger than $3.5 \mathrm{~km}$, we reset their corresponding deviation to $[0.5,0.5]$, and for the rest we reset the deviation to $\left[L_{x} / 2, L_{y} / 2\right]$. This can be interpreted as running the cross entropy in two stages. Both run for 1000 iterations: the first runs starting with a uniform distribution in $\Omega$, and the second starts with the optimum layout of the first stage as the mean value for its initial population. In the interest of simplicity, this detail is not included in the outline of Algorithm 2.

A second algorithm that is used in Algorithm 1 is the fitting of the wake-expansion coefficient $k_{\mathrm{w}}$ to the LES data. Fitting $k_{\mathrm{w}}$ is also a non-convex optimisation problem, and therefore we simply use the CE method again, but now for a scalar field. This is summarised in Algorithm 3. For this fitting, we found a number of iterations, $N_{\text {iters }}$, of 50 sufficient for good convergence.

We remark here that Algorithm 3 can in principle be used to fit more complicated relations for $k_{\mathrm{w}}$. For instance, introducing the heuristic dependence $k_{\mathrm{w}}=a+b x$ (or similar expressions), and fitting $a$ and $b$ instead of the mean value of $k_{\mathrm{w}}$, may be an interesting approach to represent the downstream development of $k_{\mathrm{w}}$ in the wind farm related to increased turbulence levels. In the current work, we did not further explore this type of parameterisations of $k_{\mathrm{w}}$, as a sim-
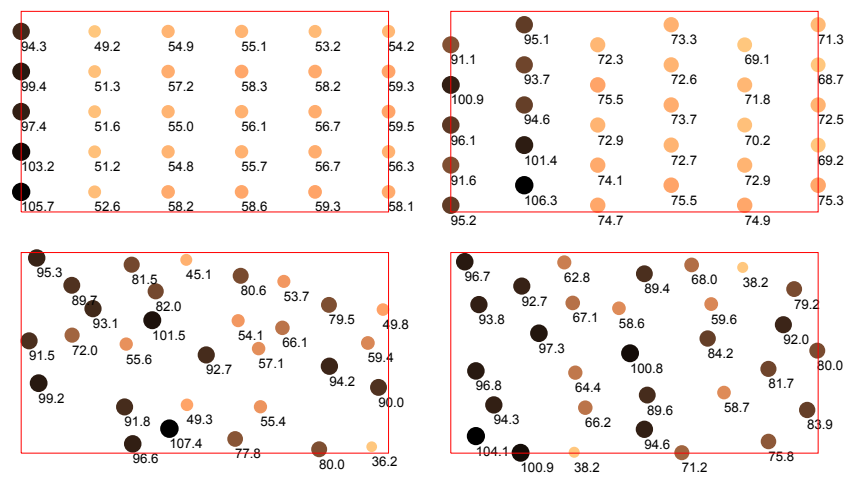

Figure 3. Layout and relative turbine power output for four of the cases listed in Table 2. Relative power results are obtained from large-eddy simulations. Turbine locations are marked with coloured disk: size and colour scale by relative power. Plot boundary (red line) corresponds to boundaries of domain $\Omega$ (see Fig. 1).

ple fit of the mean value already leads to very satisfactory results (see next section).

Finally, we remark that the CE method is a global optimisation method. However, its convergence to the global optimum in a finite number of iterations can only be formally proven for some specific conditions; in practice, convergence depends on a number heuristic choices and is difficult to formally prove (see, e.g., Rubinstein and Kroese, 2013, for details). In fact, this is a disadvantage that all global optimisation methods share. However, the main advantage of using a global method is the fact that the algorithm does not get trapped in local optimums that easily. Moreover, the disadvantage of the high number of function evaluations required for such global methods to work well is not really an issue, as Jensen-model evaluations are extremely cheap. In fact, as further discussed below, the main cost in our overall hybrid method remains associated with performing the LES.

\section{Results}

In the current section, optimisation results are discussed. First of all, in Sect. 3.1, the initial LES database for calibration of the Jensen model is constructed. Next, optimisation results of the Jensen only model are discussed in Sect. 3.2. Subsequently, hybrid Jensen-LES optimisation results are presented in Sect. 3.3. Finally, optimisation for multiple wind directions is discussed in Sect. 3.4.

\subsection{Set-up of LES database for initial calibration}

A first step in Algorithm 2 is the generation of a LES database that is a starting point for the calibration of the Jensen model. Here we choose a mix of staggered, aligned, and randomly generated layouts. An overview of the different cases and their generated power is provided in Table 2. We normalise all results with the power output of a "wake- 
Table 2. Large-eddy simulation results for different wind-farm layouts. Power output normalised with respect to total power of a wind-farm consisting of "first-row" turbines. Average LES power is $69.97 \%$.

\begin{tabular}{llr}
\hline Case no. & Description & Relative wind-farm power \\
\hline 1 & Aligned with $5 D \times 5 D$ spacing & $51.81 \%$ \\
2 & Aligned with $6 D \times 5 D$ spacing & $56.76 \%$ \\
3 & Aligned with $7 D \times 5 D$ spacing & $60.80 \%$ \\
4 & Aligned with $8 D \times 5 D$ spacing & $64.36 \%$ \\
5 & Staggered with $8 D \times 5 D$ spacing & $83.60 \%$ \\
6 & Gradually staggered with $8 D$ spacing & $87.40 \%$ \\
7 & Randomly generated with $d_{\min }=2 D$ & $79.28 \%$ \\
8 & Randomly generated with $d_{\min }=3 D$ & $76.16 \%$ \\
9 & Randomly generated with $d_{\min }=4 D$ & $78.66 \%$ \\
10 & Randomly generated with $d_{\min }=5 D$ & $80.49 \%$ \\
\hline
\end{tabular}

less" wind farm, i.e. a wind farm consisting of turbines that all have undisturbed inflow. In order to normalise all LES results in the same way, we use the averaged power output of turbines located in the first row of the aligned and staggered layouts and multiply it by $N_{\mathrm{t}}(=30)$ to find the "wakeless" wind-farm output. We then state every wind-farm power output as a percentage of this "wakeless" wind-farm output. When looking at the results of Table 2 it is apparent that the aligned cases perform quite poorly in terms of relative power output and considerably worse than the staggered cases, but they also perform worse than any of the random layouts that we investigated.

In Fig. 3 the layout and relative power output of individual turbines are shown for an aligned and a staggered layout as well as for two of the random layouts. Wind direction is always from left to right. First of all, we remark that there is still considerable variability at turbine level that is due to the limited averaging period of $3600 / u_{*} \mathrm{~s}$. As shown in Fig. 2b, variability in the turbine power average is in the order of $\pm 5 \%$ or more, and this is in line with the variability observed in the first row of Fig. 3. We verified that first-row turbine averages all converge to a relative power of $100 \%$ when averages of up to $15 / u_{*} \mathrm{~h}$ are used. Finally, we note that the accumulated farm power is much better converged.

\subsection{Comparison of Jensen model and LES results}

Without access to reference results that can serve to tune $k_{\mathrm{w}}$ in the Jensen model, it is possible to resort to Eq. (4) to determine $k_{\mathrm{w}}$. Using this equation for our simulation set-up leads to

$k_{\mathrm{w}}=\frac{0.41}{\ln (100 / 0.1)}=0.060$.

Here we briefly compare the Jensen model using this value with LES results. To do so, we use the 10 layouts presented in Table 2.

A comparison of flow fields as generated by the Jensen model and LES is shown in Fig. 4. It is seen that the aver-
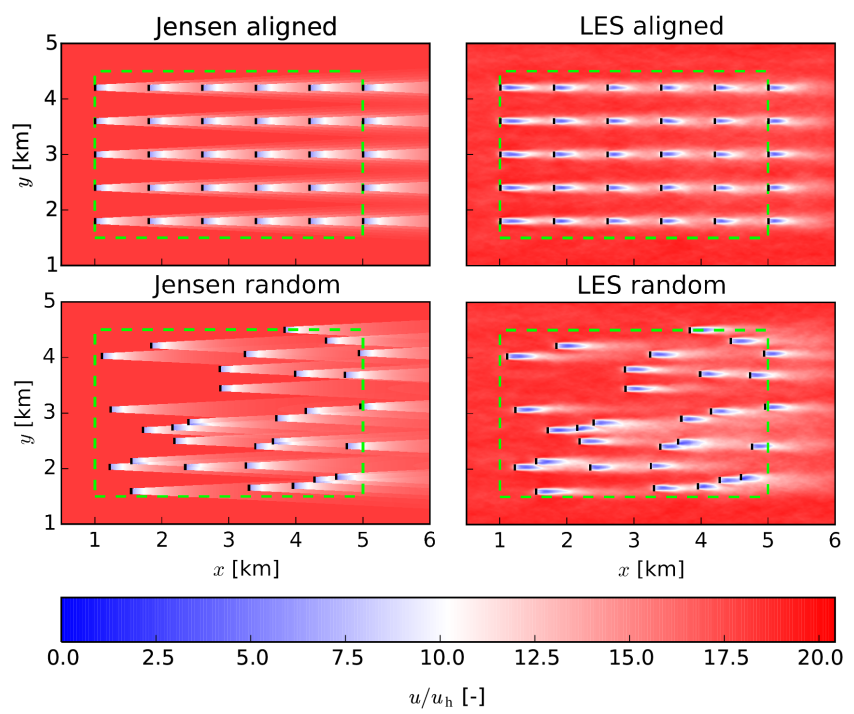

Figure 4. Comparison of Jensen model and LES for an aligned and random layout.

aged flow data of LES are much smoother as a result of turbulent mixing. In contrast, in the Jensen model, wakes have a sharp boundary, also leading to sharply marked overlap regions. Note that mirror wakes also occur more downstream in the farm. Some features are not represented at all by the Jensen model. For instance, in the random layout, it is seen that side-by-side wakes can influence each other. Such behaviour is not parameterised in the Jensen model.

However, the most relevant property from a power optimisation point of view is the total error in the predicted power. In Table 3, the average power output from LES and the Jensen model is compared. It is seen that the Jensen model using $k_{\mathrm{w}}=0.060$ is very accurate for some cases, but not so for others. In particular, the cases that have a higher relative power extraction are generally predicted worse by the Jensen model, than the cases with a lower relative power (the most prominent exception is Case 6). Another trend is that 
Table 3. Comparing outputs of LES and the Jensen wake model with $k_{\mathrm{W}}=0.060$.

\begin{tabular}{lccc}
\hline Case & $\begin{array}{c}\text { Relative } \\
\text { power } \\
\text { (LES) }\end{array}$ & $\begin{array}{c}\text { Relative } \\
\text { power } \\
\text { (Jensen } \\
\text { model) }\end{array}$ & Error \\
& & & \\
\hline Aligned 5D $\times 6 D$ & $51.21 \%$ & $52.30 \%$ & $-1.09 \%$ \\
Aligned 6D $\times 6 D$ & $55.93 \%$ & $57.98 \%$ & $-2.05 \%$ \\
Aligned 7D $\times 6 D$ & $60.13 \%$ & $62.88 \%$ & $-2.75 \%$ \\
Aligned 8D $\times 6 D$ & $63.34 \%$ & $66.83 \%$ & $-3.50 \%$ \\
Staggered $8 D$ & $82.33 \%$ & $86.81 \%$ & $-4.48 \%$ \\
Gradually staggered $8 D$ & $85.77 \%$ & $89.18 \%$ & $-3.41 \%$ \\
Random1 & $78.29 \%$ & $85.20 \%$ & $-6.91 \%$ \\
Random2 & $74.77 \%$ & $82.30 \%$ & $-7.53 \%$ \\
Random3 & $77.96 \%$ & $84.95 \%$ & $-6.99 \%$ \\
Random4 & $79.17 \%$ & $84.04 \%$ & $-4.87 \%$ \\
\hline
\end{tabular}

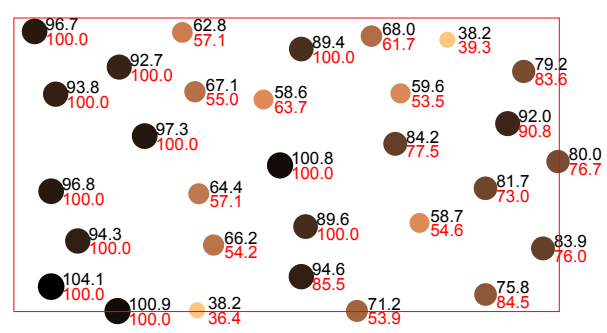

Figure 5. Comparing the wind-turbine power generation obtained from LES data (black numbers) and Jensen model (red numbers). Turbine locations are marked with coloured disk: size and colour scale by relative power. Plot boundary (red box) corresponds to boundaries of domain $\Omega$ (see Fig. 1).

the regular cases are better predicted than the irregular cases. However, in the context of optimisation, it is not important for the Jensen model to be accurate over a wide range of different layouts. Far away from the optimal layout, the required accuracy can be allowed to be considerably lower than close to the optimum. In this sense, Algorithm 2 gradually adapts the Jensen model through its wake-expansion coefficient to better fit more performing layouts.

Finally, when looking at turbine level in Fig. 5 for one of the random layouts (i.e. Case 10), it is seen that errors at the turbine level are much larger than the error on the accumulated power reported in Table 3. Again, from an optimisation point of view, this is less of an issue as long as a coupled approach in combination with LES is used to adapt the model and verify the overall results close to the optimum. We further notice here that the statistical errors on the averaged turbine power output from LES are still significant due to the limited time of averaging (in the order of $5 \%$ - see discussion in Sect. 3.1).

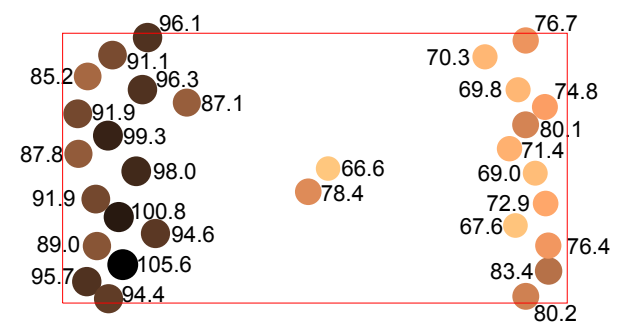

Figure 6. Optimal layout and relative power for a single wind direction obtained after iteration 1. Relative power results are obtained from large-eddy simulations. Turbine locations are marked with coloured disk: size and colour scale by relative power. Plot boundary (red line) corresponds to boundaries of domain $\Omega$ (see Fig. 1).

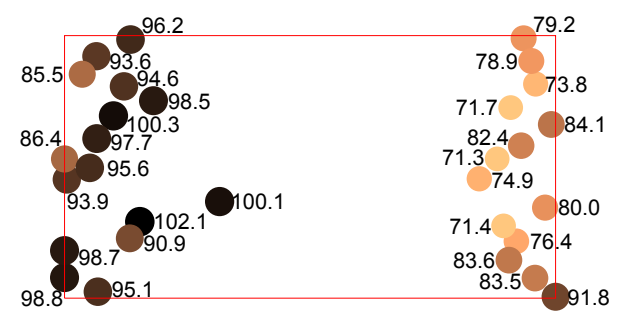

Figure 7. Optimal layout and relative power for a single wind direction obtained after iteration 2. Relative power results are obtained from large-eddy simulations. Turbine locations are marked with coloured disk: size and colour scale by relative power. Plot boundary (red line) corresponds to boundaries of domain $\Omega$ (see Fig. 1).

\subsection{Hybrid Jensen-LES optimisation}

Using Algorithm 2, we now optimise the wind-farm layout with a single constant wind direction given the set-up in Fig. 1 and wind coming from the left. Strictly speaking, this corresponds to the situation where $f_{\mathrm{p}}(\boldsymbol{\mu})$ in Eq. (2) corresponds to a Dirac delta function centred around an eastern wind direction, so that the integral over atmospheric conditions in Eq. (2) drops out. Optimisation over different wind directions is briefly discussed in Sect. 3.4. For the single wind-direction case considered here, only three outer iterations are required in the algorithm to converge to an optimal layout and optimally tuned Jensen model. Intermediate results of these iterations are discussed below.

In iteration 1, we start Algorithm 2 with the initial cases shown in Table 2. Using these 10 cases, we use Algorithm 4 to optimise the value of $k_{\mathrm{w}}$, finding a value of $k_{\mathrm{w}}=0.055$. Subsequently, this value is used to optimise the layout using Algorithm 3. The resulting optimal layout is shown in Fig. 6. Table 4 summarises the relative LES and Jensen power, as well as errors for the 10 initial training cases and for the newly obtained optimal layout. The relative power generated by the newly found optimum corresponds to $90.5 \%$ (evaluated using the LES), but the error with the Jensen model is still noticeable, i.e. $-2.62 \%$. 
Table 4. Iteration 1: comparing outputs of LES and Jensen wake model with $k_{\mathrm{W}}=0.055$.

\begin{tabular}{lccr}
\hline Case & $\begin{array}{c}\text { Relative } \\
\text { power } \\
\text { (LES) }\end{array}$ & $\begin{array}{c}\text { Relative } \\
\text { power } \\
\text { (Jensen } \\
\text { model) }\end{array}$ & Error \\
& & & \\
\hline Aligned $5 D \times 6 D$ & $51.21 \%$ & $49.59 \%$ & $1.62 \%$ \\
Aligned $6 D \times 6 D$ & $55.93 \%$ & $55.40 \%$ & $0.53 \%$ \\
Aligned 7D $\times 6 D$ & $60.13 \%$ & $60.17 \%$ & $-0.04 \%$ \\
Aligned $8 D \times 6 D$ & $63.34 \%$ & $64.22 \%$ & $-0.88 \%$ \\
Staggered $8 D$ & $82.33 \%$ & $85.78 \%$ & $-3.46 \%$ \\
Gradually staggered $8 D$ & $85.77 \%$ & $92.27 \%$ & $-6.50 \%$ \\
Random1 & $78.29 \%$ & $84.52 \%$ & $-6.23 \%$ \\
Random2 & $74.77 \%$ & $81.23 \%$ & $-6.46 \%$ \\
Random3 & $77.96 \%$ & $84.51 \%$ & $-6.55 \%$ \\
Random4 & $79.17 \%$ & $83.27 \%$ & $-4.10 \%$ \\
\hline Optimum iter. 1 & $90.51 \%$ & $93.13 \%$ & $-2.62 \%$ \\
\hline
\end{tabular}

Table 5. Iteration 2: comparing outputs of LES and Jensen wake model with $k_{\mathrm{W}}=0.036$.

\begin{tabular}{lrrr}
\hline Case & $\begin{array}{c}\text { Relative } \\
\text { power } \\
\text { (LES) }\end{array}$ & $\begin{array}{c}\text { Relative } \\
\text { power } \\
\text { (Jensen } \\
\text { model) }\end{array}$ & Error \\
& & & \\
\hline Staggered 8D & $82.33 \%$ & $79.01 \%$ & $3.32 \%$ \\
Gradually staggered 8D & $85.77 \%$ & $93.06 \%$ & $-7.29 \%$ \\
Random1 & $78.29 \%$ & $81.81 \%$ & $-3.52 \%$ \\
Random3 & $77.96 \%$ & $82.27 \%$ & $-4.31 \%$ \\
Random4 & $79.17 \%$ & $77.80 \%$ & $1.37 \%$ \\
Random5 & $79.54 \%$ & $82.06 \%$ & $-2.52 \%$ \\
Random6 & $76.01 \%$ & $78.67 \%$ & $-2.65 \%$ \\
Random7 & $80.96 \%$ & $83.50 \%$ & $-2.54 \%$ \\
Random8 & $76.25 \%$ & $78.04 \%$ & $-1.79 \%$ \\
Optimum iter. 1 & $90.51 \%$ & $90.17 \%$ & $0.34 \%$ \\
\hline Optimum iter. 2 & $92.04 \%$ & $91.88 \%$ & $0.17 \%$ \\
\hline
\end{tabular}

In iteration 2, we add optimal layout 1 and four additional random layouts to the LES database and remove the 5 layouts with lowest relative power. Using Algorithm 4, we find a new value $k_{\mathrm{w}}=0.036$ that best fits the Jensen model to the LES data. Subsequently, using Algorithm 3, a new optimal layout is found, which is shown in Fig. 7. Furthermore, an overview of relative powers from LES and Jensen is shown in Table 5. It is seen that the new optimal layout leads to a relative power of $92.8 \%$ (evaluated using LES), but in contrast to the first iteration, the error with the Jensen model remains now limited to $0.17 \%$.

As can be seen, the two optimum layouts, although obtained using different values of $k_{\mathrm{w}}$, have the same general structure.
Table 6. Optimum values of $k_{\mathrm{W}}$ obtained in different iterations of Algorithm 2.

\begin{tabular}{lcc}
\hline $\begin{array}{l}\text { Iteration } \\
\text { no. }\end{array}$ & $\begin{array}{c}\text { optimum } \\
k_{\mathrm{W}}\end{array}$ & $\begin{array}{c}\text { Relative LES } \\
\text { power of } \\
\text { corresponding } \\
\text { optimum } \\
\text { layout }\end{array}$ \\
\hline 1 & $0.055 \%$ & $90.51 \%$ \\
2 & $0.036 \%$ & $92.04 \%$ \\
3 & $0.036 \%$ & $\mathrm{NA}$ \\
\hline
\end{tabular}

NA: not available.

In iteration 3, we repeat the procedure a third time and find (almost) the same value for $k_{\mathrm{w}}$. Only the fourth digit differs, and the resulting new optimal layout remains the same. In fact, we observed that up to changes in the second digit, the value of $k_{\mathrm{w}}$ does not significantly influence the optimal layout. Finally, the error between the Jensen model and the LES is below $1 \%$, which corresponds roughly to the statistical averaging accuracy of the LES. We conclude that the algorithm is converged.

After initial set-up of the LES database, each main optimisation step requires $2.5 \times 10^{6}$ Jensen evaluations per iteration and five LES evaluations. Wall time for the Jensen evaluations (per iteration) corresponds roughly to $1.25 \mathrm{~h}$ on one Ivy Bridge node of the ThinKing cluster of the Flemish Supercomputer Centre. Total wall time for LES (per iteration, and excluding the precursor spin-up time - see Sect. 2.3) amounts to approximately $70 \mathrm{~h}$ on eight nodes of the Flemish Supercomputer, equivalent to 560 node hours. Even though the Jensen model is 500000 times more evaluated per iteration than LES, the total LES cost is roughly 500 times more expensive and the LES wall time is roughly 50 times longer.

Given this single wind direction, the optimal layout leads to a relative wind-farm performance of around $93 \%$ of a wakeless wind farm, which is considerably higher than a typical aligned or staggered layout. Moreover, when looking at the layout that was found in Fig. 7, it is observed that turbines are grouped into two main clusters - one at the front of the farm and one at the back of the farm, leaving a large streamwise distance in between for wake recovery. Obviously, this result is particular for a single wind direction. In the next section, we study the cases with multiple wind directions.

Finally, we remark that it is difficult to prove formal convergence of the $\mathrm{CE}$ method that we use for our optimisation (see discussion at the end of Sect. 2.4), and optimisation is terminated based on a maximum number of iterations in Algorithms 2 and 3. Therefore, we checked the dependence of our results versus the initial starting point of the optimisation. We found that a change in initial distribution leads to slight shifts in the turbine locations, but this does not significantly influence the value of the power extraction. More- 
over, we also experimented with the use of gradient-based optimisation using the CE optimum as a starting point of the gradient-based method. To this end, we employed Matlab's fmincon routine. Unfortunately, including all nonlinear distance constraints did not work (given 30 turbines, there are 435 distance constraints). Omitting these in the gradientbased method, we found that turbine locations again slightly shift, but that power increases by $0.4 \%$ only, indicating the obtained CE optimum is well converged. Overall, we find that the energy function is relatively flat near the region of optimal power production - i.e. small shifts in the turbine locations do not lead to significant changes in power output.

\subsection{Optimisation for multiple wind directions}

We now consider optimisation over a wind-direction distribution. Two cases are considered. The first corresponds to a uniform wind distribution over an angle of $\pm 7.5^{\circ}$, representing a case with a dominant wind direction. The second corresponds to a uniform wind distribution over an angle of $\pm 180^{\circ}$, representing a case without a dominant wind direction.

In order to properly represent power output over the wind distribution using the Jensen model, we sample the uniform distributions in $1.5^{\circ}$ increments, and the integral in Eq. (2) is discretised using a Riemann sum based on these intervals each with constant probability. For LES evaluations, we use a much coarser sampling: for the dominant wind-direction case we use only three directions and for the uniform $360^{\circ}$ case eight directions. The error between Jensen model and LES is only defined relying on these distinct directions. In this way, the overall computational costs related to LES remains limited compared to the additional Jensen model evaluations that are performed.

We first focus on the dominant wind-direction case and perform an optimisation using the Jensen model and $k_{\mathrm{w}}=$ 0.036 obtained in the previous section. An overview of the errors between Jensen model and LES for the optimal layout is given in Table 7. It is seen that errors are already below $2 \%$ for all directions, and therefore we do not further perform iterations using Algorithm 2 here. The overall optimal power output corresponds to $93.67 \%$, and the related layout is shown in Fig. 8. It is seen that the optimal layout for the dominant wind direction very much resembles the layout for the single wind-direction case. Turbines are again clustered in two large groups, one in front and one at the back of the wind farm.

Finally, we look at optimisation for the uniform wind distribution. Again we perform optimisation using the Jensen model and $k_{\mathrm{w}}=0.036$. An overview of the errors for the optimal layout is provided in Table 8. Also, errors are now overall relatively low, and so, for the sake of saving computational resources, we do not perform further iterations using Algorithm 2. We further find that, overall, the average power output of the optimised layout corresponds to $93.45 \%$. This

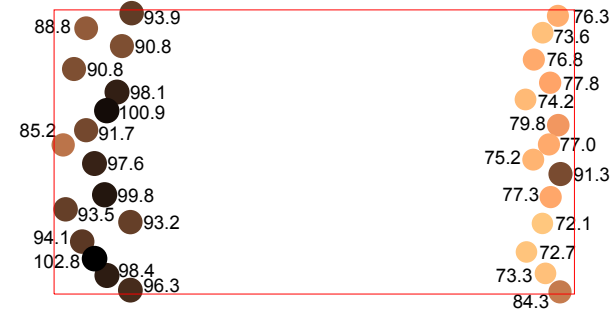

Figure 8. Optimal layout and relative power for dominant winddirection case (angle of $\pm 7.5^{\circ}$ ). Relative power results are obtained from large-eddy simulations. Turbine locations are marked with coloured disk: size and colour scale by relative power. Plot boundary (red box) corresponds to boundaries of domain $\Omega$ (see Fig. 1).

Table 7. Dominant wind-direction case - evaluation of optimal layout. Relative power for three different wind directions comparing outputs of LES and Jensen wake model with $k_{\mathrm{W}}=0.036$.

\begin{tabular}{|c|c|c|c|}
\hline $\begin{array}{l}\text { Wind direction } \\
\text { (degrees) }\end{array}$ & $\begin{array}{c}\text { Relative } \\
\text { power } \\
\text { (LES) }\end{array}$ & $\begin{array}{c}\text { Relative } \\
\text { power } \\
\text { (Jensen } \\
\text { model) }\end{array}$ & Error \\
\hline 0 & $93.08 \%$ & $91.91 \%$ & $1.17 \%$ \\
\hline 7.5 & $94.27 \%$ & $93.21 \%$ & $1.06 \%$ \\
\hline-7.5 & $93.44 \%$ & $92.43 \%$ & $1.02 \%$ \\
\hline Average $(0,7.5,-7.5)$ & $93.67 \%$ & $92.51 \%$ & $1.08 \%$ \\
\hline Average $(-7.5,1.5,7.5)$ & - & $92.53 \%$ & - \\
\hline
\end{tabular}

compares to 71.73 and $75.25 \%$ for the aligned $8 D \times 6 D$ and for staggered layout respectively.

The optimal layout itself is shown in Fig. 9. In contrast to the layout found for the dominant wind direction, turbines are now spread out much more evenly throughout the domain. Moreover, a number of turbines, i.e. seven, are located on the domain boundary. We remark here that, for similar optimisation cases in the literature, turbines sometimes end up at the domain corners (see, e.g., Réthoré et al., 2014 or Feng and Shen, 2015), but this is not the case for all studies (e.g. Du Pont and Cagan, 2012). Currently, we are not sure whether this is possibly related to domain shape, size, and number of turbines, or whether this is related to the existence of local optimums or convergence of the optimisation method. Using a hybrid method that combines a global method with a gradient-based approach, as proposed by (Réthoré et al., 2014), and exploring a large number of optimisation starting points, may be required for studying this in more detail. This is an interesting topic for further research.

\section{Conclusions}

In the current work, we proposed a hybrid Jensen-LES approach for layout optimisation of wind farms. The Jensen 


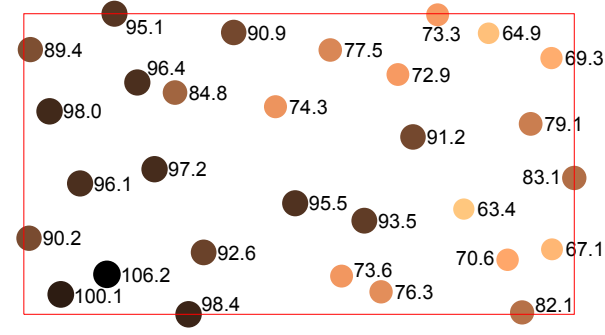

Figure 9. Optimal layout and relative power for the uniform winddirection case (angle of $\pm 180^{\circ}$ ). Relative power results are obtained from large-eddy simulations. Turbine locations are marked with coloured disk: size and colour scale by relative power. Plot boundary (red box) corresponds to boundaries of domain $\Omega$ (see Fig. 1).

Table 8. Uniform $360^{\circ}$ case - evaluation of optimal layout. Relative power for eight different wind directions, comparing outputs of LES and Jensen wake model with $k_{\mathrm{W}}=0.036$.

\begin{tabular}{lcrr}
\hline $\begin{array}{l}\text { Wind direction } \\
\text { (degrees) }\end{array}$ & $\begin{array}{c}\text { Relative } \\
\text { power } \\
\text { (LES) }\end{array}$ & $\begin{array}{c}\text { Relative } \\
\text { power } \\
\text { (Jensen } \\
\text { model) }\end{array}$ & Error \\
\hline 0 & $87.11 \%$ & $89.16 \%$ & $-2.05 \%$ \\
45 & $96.08 \%$ & $96.06 \%$ & $0.03 \%$ \\
90 & $94.75 \%$ & $94.37 \%$ & $0.39 \%$ \\
135 & $96.27 \%$ & $95.27 \%$ & $1.00 \%$ \\
180 & $87.97 \%$ & $89.41 \%$ & $-1.45 \%$ \\
-135 & $97.36 \%$ & $96.35 \%$ & $1.01 \%$ \\
-90 & $94.59 \%$ & $94.37 \%$ & $0.23 \%$ \\
-45 & $95.46 \%$ & $94.91 \%$ & $0.55 \%$ \\
Average & $93.45 \%$ & $93.55 \%$ & $-0.10 \%$ \\
\hline
\end{tabular}

model is a wake model that is sufficiently fast to allow, in principle, wind-farm optimisation over different wind directions and using global optimisation approaches that take into account the non-convex nature of the optimisation problem. Large-eddy simulations are much more accurate than the Jensen model, but they are by orders of magnitude too slow to be used for wind-farm layout optimisation. Therefore, we introduce a nested optimisation approach in which the Jensen model is used as a surrogate model. In the inner loop, the Jensen model is used to perform the layout optimisation, while in an outer loop, the wake-expansion coefficient in the Jensen model is adapted to better fit LES results of the gradually evolving optimal layouts.

In the current study, layout optimisation of a wind farm of 30 turbines on a $4 \mathrm{~km} \times 3 \mathrm{~km}$ area is considered. For this set-up, we found that an iterative fitting of the average wakeexpansion coefficient in the Jensen model during optimisation to be sufficient, leading to errors below $1 \%$ for the optimal layout. For larger wind-farm layouts, wind-farm areas that are more complex, or including different atmospheric stratification regimes, it may be necessary to consider a more complex parameterisation of the wake-expansion coefficient. This may include dependence of the wake-expansion coefficient on wind direction, Obukhov scale, or downstream location in the wind farm. These are topics for further research.

Finally, the layouts found for the current set-up differed greatly depending on the wind-direction scenario. In the case of a dominant wind direction, turbines were clustered together at the front and back of the wind-farm area, allowing for maximum wake recovery in between. For a $360^{\circ}$ uniformly distributed wind rose, turbines are evenly spread out over the domain. However, this is a result of optimisation of energy yield only, given a number of turbines and wind-farm area, and the effect of wake-wake and wake-boundary-layer interaction. In practice, wind-farm layout optimisation is a multidisciplinary problem that includes effects and costs of turbine loading, costs of installation, maintenance, cabling, etc. The full inclusion of a hybrid Jensen-LES model in such an optimisation framework is also an important topic of further research.

\section{Data availability}

Data sets supporting this article can be found in a figshare repository (Bokharaie et al., 2016), consisting of wind farm flow fields, power and layout data for the different cases in the current paper.

Acknowledgements. This work is supported by the European Research Council (ActiveWindFarms, ERC grant no. 306471), and the Flemish Science Foundation (grant no. G.0376.1). Pieter Bauweraerts acknowledges support from the Agency for Innovation by Science and Technology in Flanders (IWT). The computational resources and services used in this work were provided by the VSC (Flemish Supercomputer Centre), funded by the Research Foundation - Flanders (FWO) and the Flemish Government department EWI.

Edited by: J. Lundquist

Reviewed by: two anonymous referees

\section{References}

Allaerts, D. and Meyers, J.: Large eddy simulation of a large windturbine array in a conventionally neutral atmospheric boundary layer, Phys. Fluids, 27, 065108, doi:10.1063/1.4922339, 2015.

Barthelmie, R. J., Hansen, K., Frandsen, S. T., Rathmann, O., Schepers, J. G., Schlez, W., Phillips, J., Rados, K., Zervos, A., Politis, E. S., and Chaviaropoulos, P. K.: Modelling and measuring flow and wind turbine wakes in large wind farms offshore, Wind Energy, 12, 431-444, doi:10.1002/we.348, 2009.

Barthelmie, R. J., Pryor, S., Frandsen, S. T., Hansen, K. S., Schepers, J., Rados, K., Schlez, W., Neubert, A., Jensen, L., and Neckelmann, S.: Quantifying the impact of wind turbine wakes on power output at offshore wind farms, J. Atmos. Ocean. Tech., 27, 1302-1317, 2010. 
Bokharaie, V., Bauweraerts, P., and Meyers, J.: Data accompanying paper "Wind-farm layout optimisation using a hybrid Jensen - LES approach" by: Vahid S. Bokharaie, Pieter Bauweraerts and Johan Meyers, available at: https://dx.doi.org/10.6084/m9. figshare.c.3583460.v1, 2016.

Bou-Zeid, E., Meneveau, C., and Parlange, M. B.: A scaledependent Lagrangian dynamic model for large eddy simulation of complex turbulent flows, Phys. Fluids, 17, 025105, doi:10.1063/1.1839152, 2005.

Calaf, M., Meneveau, C., and Meyers, J.: Large eddy simulation study of fully developed wind-turbine array boundary layers, Phys. Fluids, 22, 025105, doi:10.1063/1.1839152, 2010.

Canuto, C., Hussaini, M. Y., Quarteroni, A., and Zang, T. A.: Spectral Methods in Fluid Dynamics, Springer, 1988.

Chen, Y., Li, H., Jin, K., and Song, Q.: Wind farm layout optimization using genetic algorithm with different hub height wind turbines, Energ. Convers. Manage., 70, 56-65, $2013 \mathrm{a}$.

Chen, Y., Li, H., Jin, K., and Song, Q.: Wind farm layout optimization using genetic algorithm with different hub height wind turbines, Energ. Convers. Manage., 70, 56-65, $2013 \mathrm{~b}$.

Chowdhury, S., Zhang, J., Messac, A., and Castillo, L.: Unrestricted wind farm layout optimization (UWFLO): Investigating key factors influencing the maximum power generation, Renew. Energ., 38, 16-30, 2012.

De Boer, P.-T., Kroese, D. P., Mannor, S., and Rubinstein, R. Y.: A tutorial on the cross-entropy method, Ann. Oper. Res., 134, 19-67, 2005.

Du Pont, B. L. and Cagan, J.: An extended pattern search approach to wind farm layout optimization, J. Mech. Design, 134, 081002 , doi:10.1115/1.4006997, 2012.

Emami, A. and Noghreh, P.: New approach on optimization in placement of wind turbines within wind farm by genetic algorithms, Renew. Energ., 35, 1559-1564, 2010.

Feng, J. and Shen, W. Z.: Solving the wind farm layout optimization problem using random search algorithm, Renew. Energ., 78, 182-192, 2015.

Fleming, P. A., Ning, A., Gebraad, P. M. O., and Dykes, K.: Wind plant system engineering through optimization of layout and yaw control, Wind Energy, 19, 329-344, 2016.

Frandsen, S.: On the wind speed reduction in the center of large clusters of wind turbines, J. Wind Eng. Ind. Aerod., 39, 251-265, 1992.

Gaumond, M., Réthoré, P.-E., Ott, S., Peña, A., Bechmann, A., and Hansen, K. S.: Evaluation of the wind direction uncertainty and its impact on wake modeling at the Horns Rev offshore wind farm, Wind Energy, 17, 1169-1178, doi:10.1002/we.1625, 2014.

Goit, J. P. and Meyers, J.: Optimal control of energy extraction in wind-farm boundary layers, J. Fluid Mech., 768, 5-50, 2015.

González, J. S., Rodriguez, A. G. G., Mora, J. C., Santos, J. R., and Payan, M. B.: Optimization of wind farm turbines layout using an evolutive algorithm, Renew. Energ., 35, 1671-1681, 2010.

Jensen, N. O.: A note on wind generator interaction, Technical Report Ris $\varnothing-M-2411$, Ris $\varnothing$ National Laboratory, Roskilde, Denmark, 1983.

Katic, I., Højstrup, J., and Jensen, N. O.: A simple model for cluster efficiency, in: European Wind Energy Association Conference and Exhibition, edited by: Palz, W. and Sesto, E., EWEC'86, Proceedings Vol. 1, 407-410, 7-9 October 1986, A. Raguzzi, Rome, 407-410, 1986.
Kusiak, A. and Song, Z.: Design of wind farm layout for maximum wind energy capture, Renew. Energ., 35, 685-694, 2010.

Lissaman, P. B. S.: Energy effectiveness of arbitrary arrays of wind turbines, J. Energy, 3, 323-328, 1979.

Marmidis, G., Lazarou, S., and Pyrgioti, E.: Optimal placement of wind turbines in a wind park using Monte Carlo simulation, Renew. Energ., 33, 1455-1460, 2008.

Mason, P. J. and Thomson, T. J.: Stochastic backscatter in largeeddy simulations of boundary layers, J. Fluid Mech., 242, 51-78, 1992.

Meyers, J. and Meneveau, C.: Large eddy simulations of large windturbine arrays in the atmospheric boundary layer, 48th AIAA Aerospace Sciences Meeting Including the New Horizons Forum and Aerospace Exposition, Orlando, Florida, AIAA 2010-827, $1-10,2010$.

Meyers, J. and Meneveau, C.: Flow visualization using momentum and energy transport tubes and applications to turbulent flow in wind farms, J. Fluid Mech., 715, 335-358, 2013.

Moeng, C.-H.: A large-eddy simulation model for the study of planetary boundary-layer turbulence, J. Atmos. Sci., 41, 2052-2062, 1984.

Munters, W., Meneveau, C., and Meyers, J.: Turbulent Inflow Precursor Method with Time-Varying Direction for Large-Eddy Simulations and Applications to Wind Farms, Bound.-Lay. Meteorol., 159, 305-328, 2016a.

Munters, W., Meneveau, C., and Meyers, J.: Shifted periodic boundary conditions for simulations of wall-bounded turbulent flows, Phys. Fluids, 28, 025112, doi:10.1063/1.4941912, 2016b.

Niayifar, A. and Porté-Agel, F.: A new analytical model for wind farm power prediction, J. Phys., 625, 012039, doi:10.1088/1742596/625/1/012039, 2015.

Porté-Agel, F., Wu, Y.-T., Lu, H., and Conzemius, R. J.: Large-eddy simulation of atmospheric boundary layer flow through wind turbines and wind farms, J. Wind Eng. Ind. Aerod., 99, 154-168, 2011.

Rathmann, O., Frandsen, S., and Barthelmie, R.: Wake modelling for intermediate and large wind farms, European Wind Energy Association (EWEA), Paper BL3.199, 2007.

Réthoré, P.-E., Fuglsang, P., Larsen, G. C., Buhl, T., Larsen, T. J., and Madsen, H. A.: TOPFARM: Multi-fidelity optimization of wind farms, Wind Energy, 17, 1797-1816, 2014.

Rubinstein, R.: The cross-entropy method for combinatorial and continuous optimization, Methodol. Comp. Appl., 1, 127-190, 1999.

Rubinstein, R. Y. and Kroese, D. P.: The cross-entropy method: a unified approach to combinatorial optimization, Monte-Carlo simulation and machine learning, Springer Science \& Business Media, 2013.

Saavedra-Moreno, B., Salcedo-Sanz, S., Paniagua-Tineo, A., Prieto, L., and Portilla-Figueras, A.: Seeding evolutionary algorithms with heuristics for optimal wind turbines positioning in wind farms, Renew. Energ., 36, 2838-2844, 2011.

Samorani, M.: The wind farm layout optimization problem, in: Handbook of Wind Power Systems, 21-38, Springer, 2013.

Smagorinsky, J.: General circulation experiments with the primitive equations: I. The basic experiment, Mon. Weather Rev., 91, 99$165,1963$. 
Spalart, P. R. and Watmuff, J. H.: Experimental and numerical study of a turbulent boundary layer with pressure gradients, J. Fluid Mech., 249, 337-371, 1993.

Stevens, R. J., Graham, J., and Meneveau, C.: A concurrent precursor inflow method for Large Eddy Simulations and applications to finite length wind farms, Renew. Energ., 68, 46-50, 2014.

Stevens, R. J., Gayme, D. F., and Meneveau, C.: Coupled wake boundary layer model of wind-farms, J. Renew. Sustain. Energ., 7, 023115, doi:10.1063/1.4915287, 2015.

Tennekes, H. and Lumley, J. L.: A first Course in Turbulence, The MIT Press, 1972.

Verstappen, R. W. C. P. and Veldman, A. E. P.: Symmetrypreserving discretization of turbulent flow, J. Comput. Phys., 187, 343-368, 2003.
Wan, C., Wang, J., Yang, G., and Zhang, X.: Optimal Micro-siting of Wind Farms by Particle Swarm Optimization, in: Proceedings of the First International Conference on Advances in Swarm Intelligence - Volume Part I, ICSI'10, 198-205, Springer-Verlag, Berlin, Heidelberg, 2010.

Wu, Y.-T. and Porté-Agel, F.: Simulation of Turbulent Flow Inside and Above Wind Farms: Model Validation and Layout Effects, Bound.-Lay. Meteorol., 146, 181-205, 2013.

Yang, X., Kang, S., and Sotiropoulos, F.: Computational study and modeling of turbine spacing effects in infinite aligned wind farms, Phys. Fluids, 24, 115107, doi:10.1063/1.4767727, 2012.

Zaaijer, M.: Great expectations for offshore wind turbines - Emulation of wind farm design to anticipate their value for customers, Ph.D. thesis, Delft University of Technology, 2013. 